TRANSACTIONS OF THE

AMERICAN MATHEMATICAL SOCIETY

Volume 235, January 1978

\title{
SOME ONE-RELATOR HOPFIAN GROUPS
}

BY

DONALD J. COLLINS

ABSTRACT. The group presented by

$$
\left(a, t ; t^{-1} a^{l} t=a^{m}\right)
$$

is non-Hopfian if $l, m \neq \pm 1$ and $\pi(l) \neq \pi(m)$, where $\pi(l)$ and $\pi(m)$ denote the sets of prime divisors of $l$ and $m$. By contrast, we prove that if $w$ is a word of the free group $F\left(a_{1}, a_{2}\right)$ which is not primitive and not a proper power, then the group

$$
\left(a_{1}, a_{2}, t ; t^{-1} w^{l} t=w^{m}\right)
$$

is Hopfian.

A group is Hopfian if every surjective endomorphism is an automorphism. The best-known example of a non-Hopfian group is probably the group

$$
G=\left(a, t ; t^{-1} a^{2} t=a^{3}\right)
$$

given by G. Baumslag and D. Solitar in [2]. In fact, Baumslag and Solitar considered all groups

$$
G(l, m)=\left(a, t ; t^{-1} a^{l} t=a^{m}\right)
$$

and we may summarise their conclusions as follows (incorporating a correction due to S. Meskin [7]):

If $|l|=1$ or $|m|=1$ or $|l|=|m|$ then $G(l, m)$ is residually finite and hence Hopfian.

Otherwise $G(l, m)$ is Hopfian if and only if $\pi(l)=\pi(m)$ (where $\pi(l), \pi(m)$ denote the sets of prime divisors of $l$ and $m$ respectively).

These results show the delicacy of the Hopfian property and this is further illustrated by the theorem of $\mathrm{G}$. Baumslag in [1] asserting that the group

$$
G(l, m, n)=\left(a, t ;\left(t^{-1} a^{l} t a^{-m}\right)^{n}=1\right)
$$

is residually finite when $n>1$ and $l$ and $m$ are coprime. In the same paper, Baumslag also states that if the one-relator group $H(1)=\left(a_{1}, a_{2}, a_{3}, \ldots\right.$; $r=1$ ), where $r$ is not a proper power, is Hopfian, then so too is the one-relator group

$$
H(n)=\left(a_{1}, a_{2}, a_{3}, \ldots ; r^{n}=1\right)
$$

Hopfian.

Received by the editors August 13, 1976.

AMS (MOS) subject classifications (1970). Primary 20E30; Secondary 20E05. 
We seek to investigate groups of the form

$$
G=\left(a_{1}, a_{2}, \ldots, a_{n}, t ; t^{-1} w^{l} t=w^{m}\right)
$$

where $w$ is a word in the free group $F$ on $a_{1}, a_{2}, \ldots, a_{n}$-and $w$ is not a proper power in $F$. Meskin proved in [7] that if $G$ is residually finite then $|l|=1$ or $|m|=1$ or $|l|=|m|$ and G. Baumslag (unpublished) has established the converse of this. In view of Baumslag's result, in determining whether or not $G$, as given above, is Hopfian we need only consider the situation in which $|l| \neq 1,|m| \neq 1$ and $|l| \neq|m|$.

If $w$ is a primitive element of $F$ then our given $G$ is isomorphic to the free product of the Baumslag-Solitar group $G(l, m)$ and a free group of rank $(n-1)$. In this situation $G$ is Hopfian if and only if $G(l, m)$ is Hopfian. For I. M. S. Dey and H. Neumann proved in [4] that the free product of two finitely generated Hopfian groups is Hopfian-and it is easy to see that a free product is non-Hopfian if one of the factors is non-Hopfian.

Our contribution is the following theorem.

ThEOREM. Let $G=\left(a_{1}, a_{2}, t ; t^{-1} w^{l} t=w^{m}\right)$ where $w$ is a word in $a_{1}, a_{2}$ that is not primitive and not a proper power in the free group $F\left(a_{1}, a_{2}\right)$. Then $G$ is Hopfian.

At present we are unable to extend our results beyond the case $n=2$.

We begin by summarising our notation. We write $G$ for the group

$$
\left(a_{1}, a_{2}, \ldots, a_{n} ; t^{-1} w^{l} t=w^{m}\right)
$$

where $w$ is a word in $a_{1}, a_{2}, \ldots, a_{n}$ that is not primitive and not a proper power in the free group $F=F\left(a_{1}, a_{2}, \ldots, a_{n}\right)$. Eventually we shall put $n=2$ but some of our results are valid for arbitrary $n$. When convenient we shall sometimes write $l[-1]=l$ and $l[1]=m$. As noted previously we may assume $|l| \neq|m|$-we shall not need to assume $|l| \neq 1$ and $|m| \neq 1$.

We write

$$
K=\left(a_{1}, a_{2}, \ldots, a_{n} ; w=1\right) \text { and } L=K *\langle t\rangle
$$

for the free product of $K$ with an infinite cyclic group on $t$. There is a canonical epimorphism $\theta: G \rightarrow L$ obtained by putting $w=1$.

We shall rely to a great extent on the theory of HNN-extensions and the corresponding normal form theorem and conjugacy lemma (see Chapter II of C. F. Miller III [8]-where HNN-extensions are called Britton extensions).

LEMMA $1 . G$ is an $H N N$-extension of $F$. In particular $F$ is a subgroup of $G$.

Proof. This is obvious. Q.E.D.

We write $u \sim v$ to mean that $u$ and $v$ are conjugate elements of $G$. We write $u \sim F$ to mean that there exists $y \in F$ such that $u \sim y$. Given $u \in G$ we 
say that $u$ is in normal form or is $t$-reduced if $u$ does not contain a subword $t^{-\varepsilon} x t^{e}$ with $x \in\left\langle w^{l[-\varepsilon]}\right\rangle$.

LEMMA 2. Let $\phi: G \rightarrow G$ be an endomorphism.

(i) Then there is an inner automorphism $\psi$ such that $w \phi \psi \in\langle w\rangle$. If $w \phi \in F$, then $\psi$ corresponds to an element of $F$.

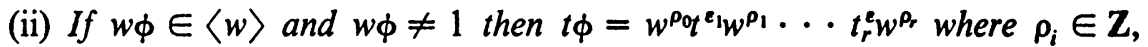
$\varepsilon_{i}= \pm 1$ and $\sum_{i=1}^{r} \varepsilon_{i}=1$.

Proof. (i) We know that $(w \phi)^{l} \sim(w \phi)^{m}$. Since $|l| \neq|m|$ the conjugacy lemma for HNN-extensions shows that $w \phi \sim F$. Without loss of generality we may assume $w \phi \in F$.

We have $(t \phi)^{-1}(w \phi)^{l}(t \phi)=(w \phi)^{m}$. Since $l \neq m, t \phi \notin F$. Let us write

$$
t=x_{0} t^{\varepsilon_{1}} x_{1} t^{\varepsilon_{2}} \cdots x_{r-1} t^{\varepsilon_{2}} x_{r}
$$

where $\varepsilon_{i}= \pm 1$ and $x_{i} \in F$. Then $x_{0}^{-1}(w \phi)^{l} x_{0} \in\left\langle w^{l\left[-\varepsilon_{0}\right\rfloor}\right\rangle$. Hence $\left\langle x_{0}^{-1}(w \phi) x_{0}\right\rangle \cap\langle w\rangle \neq 1$ giving $x_{0}^{-1}(w \phi) x_{0} \in\langle w\rangle$, since $F$ is free and $w$ is not a proper power. This proves (i).

(ii) Let $w \phi=w^{k}$ so that $(t \phi)^{-1} w^{k l}(t \phi)=w^{k m}$. The lemma follows from the fact that if $u$ is any element of $G$ and $u^{-1} w^{p} u=w^{q}$ then $u=$ $w^{\rho_{0} t^{\varepsilon_{1}}} w^{\rho_{1}} \cdots t^{e_{r}} w^{\rho_{r}}$ and $q=p(m / l)^{\sigma}$ where $\sigma=\sum_{i=1}^{r} \varepsilon_{i}$. This fact is established by induction on the number of occurrences of $t$ in the normal form of $u$ and relies heavily on the fact that if $x^{-1} w^{r} x=w^{s}$, where $x \in F$, then $r=s$ and $x \in\langle w\rangle$. (Again we do use the fact that $w$ is not a proper power.)

LEMMA 3. Let $u=t^{\varepsilon_{1}} w^{\rho_{1}} t^{\varepsilon_{2}} \cdots w^{\rho_{r-1} t^{\varepsilon_{r}}}$ be in normal form and suppose that

(i) $u^{-1} w^{l} u=w^{m}$

(ii) $G=\langle a, b, u\rangle$.

Then $u=t$, i.e. $r=1$ and $\varepsilon_{1}=1$.

Proof. We distinguish two cases.

Case 1. Suppose $l \nmid m$ and $m \nmid l$. Then $w^{l} \notin\left\langle w^{m}\right\rangle$ and $w^{m} \notin\left\langle w^{l}\right\rangle$. Since $u^{-1} w^{l} u=w^{m}$ we deduce that $\varepsilon_{1}=1=\varepsilon_{r}$.

There must exist equalities of the form

$$
t=y_{0} u^{\eta_{1} y_{1}} \cdots u^{\eta_{y}} y_{s}, \quad y_{i} \in F, \eta_{i}= \pm 1,
$$

since $G=\langle a, b, u\rangle$. Among all such we consider one with $s$ minimal-we claim that then $s=1$.

Suppose $s>1$; then there must exist $i$ such that in reducing $u^{\eta_{i-1}} y_{i-1} u^{\eta_{i}} y_{i} u^{\eta_{i+1}}$ to normal form the occurrences of $t$ in $u^{\eta_{i}}$ are eliminated. For if this were false the normal form of the right-hand side of (1) would contain at least $s$ occurrences of $t$.

The minimality of $s$ is contradicted immediately if $u^{\eta_{i-1} y_{i-1}} u^{\eta_{i}} \in F$ or 
$u^{\eta_{i}} y_{i} u^{\eta_{i+1}} \in F$. So part of $u^{\eta_{i}}$ is cancelled by $u^{\eta_{i-1}}$ and part by $u^{\eta_{i+1}}$ (strictly only the occurrences of $t$ are cancelled). Since $\varepsilon_{1}=\varepsilon_{r}$ we have $\eta_{i-1}+\eta_{i}=0$ $=\eta_{i}+\eta_{i+1}$.

Suppose $\eta_{i-1}=1$; then we may write $u=u_{1} u_{2}$ where $u_{2} y_{i-1} u_{2}^{-1}=x_{i-1} \in$ $F$ and $u_{1}^{-1} y_{i} u_{1}=x_{i} \in F$. Now in view of the form of $u$ it is clear that both $x_{i-1}$ and $x_{i}$ lie in $\langle w\rangle$. In particular $x_{i-1} x_{i}=x_{i} x_{i-1}$. Then

$$
u y_{i-1} u^{-1} y_{i} u=u_{1} x_{i-1} x_{i} u_{2}=u_{1} x_{i} x_{i-1} u_{2}=y_{i} u y_{i-1} .
$$

Again the minimality is contradicted. The argument when $\eta_{i-1}=-1$ is similar.

So $t=y_{0} u^{\eta_{1} y_{1}}$; as $u$ is in normal form we obtain $\eta_{1}=1$ and $r=0$ as required.

Case 2. Suppose $l \mid m$ or $m \mid l$. Here there are two subcases but they can be treated similarly. So suppose that $m=l m_{0}$; then $m \nmid l$ as $|l| \neq|m|$.

From the fact that $u^{-1} w^{l} u=w^{m}$ we see that $\varepsilon_{1}=1$. However $w^{m} \in\left\langle w^{l}\right\rangle$ so we must allow for the possibility that $\varepsilon_{r}=-1$.

If in fact $\varepsilon_{r}=1$ we can argue as in Case 1. So suppose $\varepsilon_{r}=-1$; we consider equations of the form

$$
t=z_{0} u^{\lambda_{1}} z_{1} \cdots u^{\lambda} z_{s}, \quad z_{i} \in F, \lambda_{i} \in \mathbf{Z}, \lambda_{i} \neq 0 .
$$

As $G=\langle a, b, u\rangle$ such equations must exist. We again want to consider such an equation with $s$ minimal.

We claim that in this event

(a) if $i>1$ and $\left.\lambda_{i}\right\rangle 0$, then $z_{i-1} \notin\left\langle w^{l}\right\rangle$;

(b) if $i<s$ and $\lambda_{i}<0$, then $z_{i} \notin\left\langle w^{l}\right\rangle$.

We note that for any $\lambda>0$ and any $j \in \mathbf{Z}, u^{-\lambda} w^{j l} u^{\lambda}=w^{j l m_{0}^{\lambda}}$. Hence, if (a) is violated then for some $i>1$

$$
u^{\lambda-1} z_{i-1} u^{\lambda}=u^{\lambda-1}+\lambda_{i} z_{i-1}^{\prime}, \text { where } z_{i-1}^{\prime}=u^{-\lambda} z_{i} u^{\lambda} \text {. }
$$

Clearly the minimality of $s$ is contradicted. Similarly if (b) is violated, then, for some $i<s$,

$$
u^{\lambda} z_{i} u^{\lambda_{1+1}}=z_{i}^{\prime} u^{\lambda_{1}+\lambda_{1+1}}
$$

which again is contradictory.

Now write $u=u_{1} u_{2} u_{1}^{-1}$ where $u_{2}$ is cyclically $t$-reduced. Since $\varepsilon_{1}=1$ and $\varepsilon_{r}=-1, u_{1} \notin F$. Also, since $u^{-1} w^{l} u=w^{m}, \Sigma_{i=1}^{r} \varepsilon_{i}=1$ so that $u_{2} \notin F$. Our equation becomes

$$
t=z_{0} u_{1} u_{2}^{\lambda_{1}} u_{1}^{-1} z_{1} \cdots u_{1} u_{2}^{\lambda} u_{1}^{-1} z_{s} .
$$

Suppose $s>1$; there cannot exist $i$ such that $u_{1} u_{2}^{\lambda} u_{1}^{-1} z_{i} u_{1} u_{2}^{\lambda+1} u_{1}^{-1} \in F$. It follows that there exists $i$ such that in reducing

$$
u_{1} u_{2}^{\lambda-1} u_{1}^{-1} z_{i-1} u_{1} u_{2}^{\lambda} u_{1}^{-1} z_{i} u_{1} u_{2}^{\lambda+1} u_{1}^{-1}
$$


to normal form, the occurrences of $t$ in $u_{1} u_{2}^{\lambda} u_{1}^{-1}$ are eliminated partly from the left and partly from the right. This means that $z_{i-1} \in\left\langle w^{l}\right\rangle$ and $z_{i} \in\left\langle w^{l}\right\rangle$. The former implies that $\lambda_{i}<0$ and the latter that $\lambda_{i}>0$.

We conclude that $t=z_{0} u_{1} u_{2}^{\lambda_{1}} u_{1}^{-1} z_{1}$ which is impossible since the right-hand side is in normal form and has at least three occurrences of $t$. Q.E.D.

COROLlARY 4. Let $\phi: G \rightarrow G$ be a surjective endomorphism such that $F \phi \subseteq F$ and $w \phi \in\langle w\rangle, w \phi \neq 1$. Then $t \phi=w^{\rho_{0} t w^{\rho_{1}}}$.

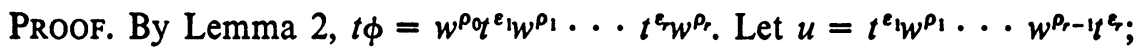
since $G=\langle a \phi, b \phi, t \phi\rangle$ and $F \phi \subseteq F$ we obtain $G=\langle a, b, u\rangle$. By Lemma 3, $u=t$. Q.E.D.

Proposition 5. Let $G=\left(a_{1}, a_{2}, \ldots, a_{n}, t ; t^{-1} w^{l} t=w^{m}\right)$ where $w$ is not primitive and not a proper power in the free group $F=F\left(a_{1}, a_{2}, \ldots, a_{n}\right)$. If $\phi$ is a surjective endomorphism such that $F \phi \subseteq F$, then $\phi$ is an automorphism.

PRoof. Certainly $w \phi \in F$ and, using Lemma 2 , we may assume $w \phi \in\langle w\rangle$. More explicitly this asserts that for some $k \in \mathbf{Z}$

$$
w\left(a_{1} \phi, a_{2} \phi, \ldots, a_{n} \phi\right)=w^{k}
$$

and this equality holds in $F$. Suppose $k \neq \pm 1$; by a theorem of G. Baumslag and A. Steinberg in [3], the rank of the group $\left\langle a_{1} \phi, a_{2} \phi, \ldots, a_{n} \phi, w\right\rangle$ is at most $(n-1)$. Then certainly the rank of $\left\langle a_{1} \phi, a_{2} \phi, \ldots, a_{n} \phi\right\rangle$ is at most $(n-1)$ and so $G$ can be generated by at most $n$ elements. This means that

$$
L=\left(a_{1}, a_{2}, \ldots, a_{n} ; w=1\right) *\langle t\rangle
$$

can be generated by at most $n$ elements. By Gruško's theorem

$$
K=\left(a_{1}, a_{2}, \ldots, a_{n} ; w=1\right)
$$

is generated by at most $(n-1)$ elements. By a theorem of W. Magnus (Corollary 5.14.2 of [5]), $K$ is a free group. By Whitehead's theorem (Theorem N.3 of Chapter 3 of [5]), $w$ is primitive in $F$. Thus $w \phi=w^{ \pm 1}$.

Now by the same argument as above, the group $\left\langle a_{1} \phi, a_{2} \phi, \ldots, a_{n} \phi\right\rangle$ is free of rank $n$ and thus $\phi$ is injective on $F$. Suppose $v \phi=1$ and $v \neq 1$. Then certainly $v \notin F$. By the normal form theorem for HNN-extensions $v$ must contain a subword $t^{-\varepsilon} z t^{\varepsilon}$ where $z \in\left\langle w^{l[-\varepsilon]}\right\rangle$. By Corollary $4, t \phi=w^{\rho_{0}} t w^{\rho_{1}}$ and so $v$ must contain a subword $t^{-\xi} t^{\ell}$ such that $y \phi=z=w^{k l[-\varepsilon]}$. Now we know that $w \phi=w^{\eta}, \eta= \pm 1$ and hence $\left(w^{\eta k l[-\varepsilon]}\right) \phi=y \phi$. As $\phi$ is injective on $F, y \in\left\langle w^{l[-\varepsilon]}\right\rangle$. Inductively, this means that $v \in F$ which is impossible. Q.E.D.

We have on the face of it proved a little more than was necessary to obtain Proposition 5. It would suffice to prove that $t \phi$ was of the form $w^{\rho_{0} t^{\varepsilon_{1}}} x_{1} \cdots x_{r-1} t^{\varepsilon_{r}} w^{\rho_{r}}$ with $\varepsilon_{1}=1=\varepsilon_{r}$. But much of Lemma 3 is needed for 
this and we have obtained additional information about automorphisms of $G$.

We now turn to the situation where we must impose the additional hypothesis that $n=2$. This is done in order to deal with the situation where $F \phi \& F$ (even allowing for inner automorphisms). We shall show that, in these circumstances, $\phi$ cannot be surjective.

Given $u_{1}, u_{2}, u_{3} \notin F$ we call $\left(u_{1}, u_{2}, u_{3}\right)$ a reduction triple if in the process of reducing $u_{1} u_{2} u_{3}$ to normal form, the occurrences of $t$ in $u_{2}$ are eliminated.

Lemma 6. Let $u_{i} \notin F, i=1,2, \ldots, s$, suppose that $u_{1} u_{2} \cdots u_{s} \sim F$. Then, for some $i,\left(u_{i-1}, u_{i}, u_{i+1}\right)$ is a reduction triple-including the possibility that $i=1$ or $i=s$ in which case $i-1$ and $i+1$ are to be interpreted modulo $s$.

PROof. If the conclusion were false the cyclically $t$-reduced form of $u_{1} u_{2} \cdots u_{s}$ would not be free of occurrences of $t$. Q.E.D.

We begin with the situation in which $a_{1} \phi \notin F$ and $a_{2} \phi \in F$. Firstly we require a rather technical lemma.

LEMma 7. Let $u \notin F$ and $y \in F$ be such that $\left(u^{e}, y^{r} u^{\eta}, y^{s} u^{\nu}\right)$, where $r, s \in \mathbf{Z}$ and $\varepsilon, \eta, \nu= \pm 1$ is a reduction triple. Then the following possibilities occur:

(i) (a) $\varepsilon+\eta=0$ and $y \theta=1$ or

(b) $\eta+\nu=0$ and $y \theta=1$,

(ii) (a) $\varepsilon=\eta$, and $u^{\varepsilon} \theta=(y \theta)^{-r}$ or

(b) $\eta=\nu$, and $u^{\eta} \theta=(\nu \theta)^{-s,}$

(iii) $\varepsilon=\eta=\nu$ and $u^{\varepsilon}=u_{0} x u_{0}^{-1} y^{-r}$, for some $x \in F$ and some $u_{0}$.

(Recall that $\theta: G \rightarrow L$ is the canonical epimorphism described at the outset.)

PROOF. It is clear that either $u^{y} y^{r} u^{\eta}$ or $u^{\eta} y^{s} u^{\prime}$ is not in normal form.

Suppose the former occurs; if $\varepsilon+\eta=0$ then $y^{r} \sim\langle w\rangle$ (in $F$ ). This gives $(y \theta)^{r}=1$. Since $w$ is not a proper power, $K$ is torsion free (see [5]). Thus $y \theta=1$ and (i)(a) holds.

Let $\varepsilon=\eta=1$; if in fact $u y^{r} u \in F$ then analysis of the reduction of the $u y^{r} u$ to normal form gives rise to a system of equations in the following way. Let $u=x_{0} t^{\kappa_{1}} x_{1} \cdots t^{\kappa} x_{p}$. Then

$$
\begin{gathered}
x_{p} y^{r} x_{0}=w^{\left.i_{p} l \kappa_{p}\right]}, \quad \kappa_{p}+\kappa_{1}=0, \\
x_{p-1} w^{i_{p} l\left[-\kappa_{p}\right]} x_{1}=w^{\left.i_{p-1} l l \kappa_{p-1}\right]}, \quad \kappa_{p-1}+\kappa_{2}=0, \\
\vdots \\
x_{1} w^{\left.i_{2} l-\kappa_{2}\right]} x_{p-1}=w^{i_{1} l\left[\kappa_{1}\right]}, \quad \kappa_{1}+\kappa_{p}=0,
\end{gathered}
$$

where the group equations hold in $F$.

Now clearly $2\left(\kappa_{1}+\cdots+\kappa_{p}\right)=0$ and so $p=2 q, q \in \mathbf{Z}$. Then among the 
group equations there occurs

$$
x_{q} w^{i_{q+1} l\left[-\kappa_{q+1}{ }^{1}\right.} w_{q}=w^{i_{q} l\left[\kappa_{q}\right]} .
$$

Clearly $\left(x_{q} \theta\right)^{2}=1$ and so $x_{q} \theta=1$, since $K$ is torsion free.

Let $u_{0}=x_{0} t^{\kappa_{1}} x_{1} \cdots x_{q-1} t^{\kappa_{q}}$. The above equations imply that

$$
x_{q} t^{\kappa_{q}+1} \cdots t^{\kappa_{p}} x_{p}=x_{q} w^{-i_{q+1} l l-\kappa_{q+1} l^{l}} u_{0}^{-1} y^{-r}
$$

and hence that $u=u_{0} \hat{x}_{q} u_{0}^{-1} y^{-r}$, where $\hat{x}_{q}=x_{q} w^{-i_{q+1} l\left[-x_{q}+1\right]}$. But clearly $\hat{x}_{q} \theta$ $=1$ and so $u \theta=(y \theta)^{-r}$. The argument is similar when $\varepsilon=-1$ and we have (ii)(a).

So suppose that $u^{e} v^{r} u^{\eta} \notin F$. Then $u^{\eta} y^{s} u^{\nu}$ is not in normal form; if $\eta+\nu=0$ we have (i)(b). So assume $\varepsilon=\eta=\nu$. If $u^{\eta} y^{s} u^{\nu} \in F$ we obtain (ii)(b). Otherwise, taking $\varepsilon=1$, we obtain two systems of equations, viz.

$$
\begin{aligned}
& x_{p} y^{r} x_{0}=w^{i}\left[\kappa_{p}\right], \quad \kappa_{p}+\kappa_{1}=0, \\
& x_{c} w^{i_{c+1} l\left[-\kappa_{c+1}\right]} x_{p-c}=w^{\left.i_{c} l \kappa_{c}\right]}, \quad \kappa_{c}+\kappa_{p+1-c}=0,
\end{aligned}
$$

from reduction in $u y^{r} u$, and

$$
\begin{gathered}
x_{p} y^{s} x_{0}=w^{\left.k_{p} l k_{p}\right]}, \quad \kappa_{p}+\kappa_{1}=0 \\
\vdots \\
x_{p+2-c} w^{k_{p+3-c} l\left[k_{p+3-c}\right]} x_{c-2}=w^{k_{p+2-c} l\left[\kappa_{p+2-c}\right]}, \quad \kappa_{p+2-c}+\kappa_{c-1}=0,
\end{gathered}
$$

from reduction in $u y^{s} u$.

As before $2\left(\kappa_{1}+\cdots+\kappa_{p}\right)=0$ and $p=2 q$. It is easy to see that $c<q+$ 1 or $c=q+1$ or $p+2-c<q+1$. If $c<q+1$ we obtain $u \theta=(y \theta)^{-r}$ and if $p+2-c<q+1$ we obtain $u \theta=(\nu \theta)^{-s}$, arguing as above.

Let $c=q+1$; then we still have $u=u_{0} \hat{x}_{q} u_{0}^{-1} y^{-r}$ by substituting appropriately. We cannot, however, conclude that $\hat{x}_{q} \theta=1$; but we have (iii). A similar argument is given when $\varepsilon=-1$.

LEMMA 8. Let $u \notin F, y \in F$ be such that there is a word $z\left(a_{1}, a_{2}\right)$ such that $z(u, y) \sim F$. Then for any $g \in G,\langle u, y, g\rangle \neq G$.

Proof. Clearly we may assume that $z\left(a_{1}, a_{2}\right)$ is cyclically reduced. Rather less obviously we may assume that $z\left(a_{1}, a_{2}\right)$ is not a proper power. For suppose $z\left(a_{1}, a_{2}\right)=z_{0}\left(a_{1}, a_{2}\right)^{p}$. Then $z_{0}(u, y)^{p} \sim F$; we claim $z_{0}(u, y) \sim F$. Suppose not; then $z_{0}(u, y)$ can be expressed as $v_{1} v_{2} v_{1}^{-1}$ where $v_{2}$ is cyclically $t$-reduced, $v_{2} \notin F$. But then $z_{0}(u, y)^{p}=v_{1} v_{2}^{p} v_{1}^{-1}$ and $v_{2}^{p} \nsim F$.

Let us write $z\left(a_{1}, a_{2}\right)=a_{1}^{\varepsilon_{1}} a_{2}^{r_{1}} a_{1}^{e_{2}} a_{2}^{r_{2}} \cdots a_{1}^{\gamma_{1}} a_{2}^{r_{f}}$ where $\varepsilon_{i}= \pm 1, r_{f} \neq 0$, and $r_{i} \neq 0$ if $\varepsilon_{i}+\varepsilon_{i+1}=0$. Then we have 


$$
u^{\varepsilon_{1}} y^{r_{1}} u^{\varepsilon_{2}} y^{r_{2}} \cdots u^{\varepsilon_{y} y^{r_{f}}} \sim F .
$$

By Lemma 5, there is reduction triple $\left(u^{\xi-1}, y^{r_{j-1} u^{\xi}}, y^{r_{j} u^{\xi+1}}\right)$.

We claim that either $y \theta=1$ or $u \theta \in\langle y \theta\rangle$. This is immediate if either (i) or (ii) of Lemma 7 occurs. So suppose that $\varepsilon_{j-1}=\varepsilon_{j}=\varepsilon_{j+1}=1$ and $u=$ $u_{0} x u_{0}^{-1} y^{-r}$ where $r=r_{j-1}$. (The argument when $\varepsilon_{j-1}=\varepsilon_{j}=\varepsilon_{j+1}=-1$ is virtually identical to what follows.) Substituting in $z(u, y)$ we obtain a word of the form

$$
y^{s_{0}} u_{0} x^{e_{1}} u_{0}^{-1} y^{s_{1}} \cdots u_{0} x^{s} u_{0}^{-1} y^{s_{s}}
$$

where $s_{i}=r_{i}-\frac{1}{2}\left(\varepsilon_{i}+\varepsilon_{i+1}\right) r, i=1,2, \ldots, f, s_{0}=0$ or $r$ according as $\varepsilon_{1}=1$ or -1 , and $s_{f}=r_{f}-r$ or $r_{f}$ according as $\varepsilon_{f}=1$ or -1 .

We firstly consider the possibility that $s_{1}=s_{2}=\cdots=s_{f-1}=0$. Then $r_{i}=\frac{1}{2}\left(\varepsilon_{i}+\varepsilon_{i+1}\right) r, i=1,2, \ldots,(f-1)$. This means $\varepsilon_{1}=\varepsilon_{2}=\cdots=\varepsilon_{f}$, for otherwise there exists $i$ such that $\varepsilon_{i}+\varepsilon_{i+1}=0$ and, hence, $r_{i}=0$. Thus $r_{1}=\cdots=r_{f-1}= \pm r$. Assume $\varepsilon_{1}=1$ so that $r_{1}=r$. Then $z(u, y)=$ $u_{0} x^{f} u_{0}^{-1} y^{r_{f}-r}$. Since $z\left(a_{1}, a_{2}\right)$ is not a proper power, $r_{f} \neq r$. The fact that $y_{0} x^{f} u_{0}^{-1} y^{r_{f}-r} \sim F$ means that either $x^{f} \sim\langle w\rangle$ or $y^{r_{f}-r} \sim\langle w\rangle$ in $F$. Hence $u \theta=1$ or $v \theta=1$. If $\varepsilon_{1}=-1$ and $r_{1}=-r$, then $z(u, y)=y^{r} u_{0} x^{-f} u_{0}^{-1} y^{r}$. Since $z\left(a_{1}, a_{2}\right)$ is not a proper power, $r_{f} \neq-r$. As above we obtain $u \theta=1$ or $y \theta=1$.

Now we may suppose that some $s_{i} \neq 0, i=1,2, \ldots, f-1$. This means that $z(u, y)$ is equal after free cancellation to a word of the form

$$
y^{s_{0}} u_{0} x^{\lambda_{1}} u_{0}^{-1} y^{t_{1}} \cdots y^{t_{q}-1} u_{0} x^{\lambda_{q}} u_{0}^{-1} y^{s_{f}}
$$

where $\lambda_{i}, t_{i} \in \mathbf{Z}, q \geqslant 2, t_{i} \neq 0, i=1,2, \ldots, f, \lambda_{1}$ has the same sign as $\varepsilon_{1}$, and $\lambda_{q}$ the same sign as $\varepsilon_{f}$. Note that the initial and terminal powers of $y$ are unchanged. Since $z(u, y) \sim F$, this word is not cyclically reduced. If $u_{0} x^{\lambda} u_{0}^{-1}$ or $u_{0}^{-1} y^{t^{t}} u_{0}$, for some $i$, is not in normal form we obtain respectively $x \theta=1$ or $y \theta=1$. The claim follows in this case. If the above word is in normal form then it follows that $u_{0} y^{s_{f}+s_{0}} u_{0}^{-1}$ is not in normal form. Then of course $y \theta=1$ except when $s_{f}+s_{0}=0$ (this cannot immediately be ruled out). If $s_{f}+s_{0}=$ 0 , then $u_{0}^{-1} x^{\lambda_{q}+\lambda_{1}} u_{0}$ is not in normal form. If $\lambda_{q}+\lambda_{1} \neq 0$, this gives $x \theta=1$ and we are all right. So assume $\lambda_{q}+\lambda_{1}=0$. Then of course $\varepsilon_{1}$ and $\varepsilon_{f}$ are of opposite sign. If $\varepsilon_{1}=1$ and $\varepsilon_{f}=-1$, then $s_{0}=0$ and $s_{f}=r_{f}$ so that $r_{f}=0$. This is contradictory. If $\varepsilon_{1}=-1$ and $\varepsilon_{f}=1$, then $s_{0}=r$ and $s_{f}=r_{f}-r$ so again $r_{f}=0$ and we have a contradiction.

We can now show that $\langle u, y, g\rangle \neq G$. If $\langle u, y, g\rangle=G$, then $\langle u \theta, y \theta, g \theta\rangle$ $=L$. This means $L$ is generated by at most two elements. By our usual argument, this contradicts the fact that $w$ is not primitive.

Proposition 9. Let $\phi: G \rightarrow G$ be an endomorphism such that $a \phi \notin F$ and $b \phi \in F$. Then $\phi$ is not surjective. 
Proof. If $\phi$ is surjective then $G=\langle a \phi, b \phi, t \phi\rangle$. Since $w \phi \sim\langle w\rangle$ by Lemma 2 and $w \phi=w(a \phi, b \phi)$, Lemma 8 yields a contradiction.

For any $u \in G$, let $l_{t}(u)$ denote the number of occurrences of $t$ in $u$.

Proposition 10. Let $u, v \in G, u, v \notin F$ be such that

(1) there exists a word $z\left(a_{1}, a_{2}\right)$ such that $z(u, v) \sim F$,

(2) there exists $g \in G$ such that $G=\langle u, v, g\rangle$.

Then there exist $x, y \in F$ and $u_{0} \in G$ such that $u=u_{0} x u_{0}^{-1}$ and $v=u_{0} y u_{0}^{-1}$.

ProOF. We proceed by induction on $l_{t}(u)+l_{t}(v)$. We therefore assume the proposition false and that among all pairs $u$ and $v$ satisfying the hypotheses, but not the conclusion, we have chosen a pair with $l_{t}(u)+l_{t}(v)$ minimal.

Suppose $u \sim F$, say $u=u_{0} x u_{0}^{-1}, x \in F$. Then we have

$$
u_{0} x^{m_{1}} u_{0}^{-1} v^{n_{1}} \cdots u_{0} x^{m_{1}} u_{0}^{-1} v^{m_{1}} \sim F
$$

where $z\left(a_{1}, a_{2}\right)=a_{1}^{m_{1}} a_{2}^{n_{1}} \cdots a_{1}^{m_{j}} a_{2}^{m_{j}}$. Let $\hat{v}=u_{0}^{-1} v u_{0}$ and $\hat{g}=u_{0}^{-1} g u_{0}$. Then $G=\langle x, \hat{v}, \hat{g}\rangle$ and $z(x, \hat{v}) \sim F$. By Lemma $8, \hat{v} \in F$, i.e. $v=u_{0} y u_{0}^{-1}, y \in F$. Our minimality assumption is contradicted.

The argument when we assume $v \sim F$ is similar.

So suppose $u \nsim F$ and $v \nsim F$. Let $u=u_{1} u_{2} u_{1}^{-1}$ and $v=v_{1} v_{2} v_{1}^{-1}$ be in normal form with $u_{2}$ and $v_{2}$ cyclically $t$-reduced. Let $\hat{u}=v_{1}^{-1} u_{1} u_{2} u_{1}^{-1} v_{1}$ and $\hat{g}=v_{1}^{-1} g v_{1}$. Then $G=\left\langle\hat{u}, v_{1}, \hat{g}\right\rangle$ and $z\left(\hat{u}, v_{2}\right) \sim F$. If $\hat{u}$ (equivalently $u_{1}^{-1} v_{1}$ ) is not in normal form then the induction hypothesis gives $v_{2} \sim F$ which is contradictory. So we may assume $\hat{u}$ is in normal form.

Case (i). Let $\hat{u}$ be cyclically $t$-reduced. Then, in practice, we have $u_{1}=v_{1}=$ 1, i.e. $u=\hat{u}$ and $v=v_{2}$.

So we have

$$
u^{m_{1} v^{n_{1}}} \cdots u^{m_{v} n_{y}} \sim F
$$

where $z\left(a_{1}, a_{2}\right)=a_{1}^{m_{1}} a_{2}^{n_{1}} \cdots a_{1}^{m_{j}} a_{2}^{n_{y}}$ and $u$ and $v$ are cyclically $t$-reduced. By Lemma 6 we can find a reduction triple. The following are the only possibilities:
(1) $\left(u^{e}, u^{e}, v^{\eta}\right)$,
(2) $\left(u^{e}, v^{\eta}, v^{\eta}\right)$,
(3) $\left(u^{\varepsilon}, v^{\eta}, u^{\nu}\right)$,
(4) $\left(v^{e}, u^{\eta}, v^{\nu}\right)$,

where $\varepsilon, \eta, \nu= \pm 1$.

Suppose (1) occurs; then we can write $v^{\eta}=v_{3} v_{4}$ where $u^{\varepsilon} v_{3} \in F$. Thus $l_{t}\left(u^{e} v\right)<l_{t}(v)$. Now $G=\langle u, v, g\rangle$; also we can regard $z(u, v)$ as $z\left(u, u^{-\varepsilon}\right.$ $\left.\left(u^{e} v\right)\right)$ if $\eta=1$ and as $z\left(u,\left(v u^{-e}\right) u^{e}\right)$ if $\eta=-1$. In either case we can construct a word $z^{*}\left(a_{1}, a_{2}\right)$ such that $z^{*}\left(u, u^{e} v^{\eta}\right) \sim F$. By the induction hypothesis $u \sim F$ which is contradictory. Clearly (2) is similar to (1).

Suppose (3) occurs. If $\varepsilon+\nu=0$ then either $u^{e} v^{\eta}$ or $v^{\eta} u^{\nu}$ is $t$-reduced-since 
$v$ is cyclically $t$-reduced. Suppose $v^{\eta} u^{\nu}$ is $t$-reduced. Then we can write $u^{e}=u_{5} u_{6}$ where $u_{6} v^{\eta} \in F$. Then $l_{t}\left(u^{\varepsilon} v^{\eta}\right)\left\langle l_{t}(u)\right.$ and $G=\left\langle u^{\varepsilon} v^{\eta}, v, g\right\rangle$. Also we can transform $z(u, v)$ into $z^{*}\left(u^{e} v^{\eta}, v\right)$ and deduce that $v \sim F$ by the induction hypothesis. The alternative case is similar and so too is (4).

Case (ii). Suppose that $\hat{u}$ is not cyclically $t$-reduced. Write $u_{3}=v_{1}^{-1} u_{1}$; thus $\hat{u}=u_{3} u_{2} u_{3}^{-1}$. We have

$$
u_{3} u_{2}^{m_{1}} u_{3}^{-1} v_{2}^{n_{1}} \cdots u_{3} u_{2}^{m_{1}} u_{3}^{-1} v_{2}^{n_{y}} \sim F
$$

and Lemma 6 ensures the existence of a reduction triple. The possibilities are:

(1) $\left(u_{2}^{e}, u_{3}^{-1}, v_{2}^{\eta}\right)$,

(2) $\left(v_{2}^{\eta}, v_{2}^{\eta}, u_{3}\right)$,

(3) $\left(u_{3}^{-1}, v_{2}^{\eta}, u_{3}\right)$,

(4) $\left(u_{3}^{-1}, v_{2}^{\eta}, v_{2}^{\eta}\right)$,

(5) $\left(v_{2}^{\eta}, u_{3}, u_{2}^{e}\right)$.

We examine (1) in detail. Here we must have $v_{2}^{\eta}=v_{3} v_{4}$ where $u_{3}^{-1} v_{3} \in F$; write $x=u_{3}^{-1} v_{3}$. Then $z\left(x^{-1} u_{4} x,\left(v_{4} v_{3}\right)^{\eta}\right) \sim F$ and $G=$ $\left\langle x^{-1} u_{4} x,\left(v_{4} v_{3}\right)^{\eta}, v_{3}^{-1} \hat{g} v_{3}\right\rangle$. Since $l_{t}\left(x^{-1} u_{4} x\right)<l_{t}(\hat{u})$ we obtain $\left(v_{4} v_{3}\right)^{\eta} \sim F$ and hence $v_{2} \sim F$.

The remaining cases are dealt with in a similar manner and the proof is complete. Q.E.D.

We come finally to the proof of our main theorem.

Proof of The Theorem. Let $\phi$ be a surjective endomorphism. By Proposition 9, either $\left\langle a_{1} \phi, a_{2} \phi\right\rangle \subseteq F$ or $a_{1} \phi \notin F$ and $a_{2} \phi \notin F$. If $\left\langle a_{1} \phi, a_{2} \phi\right\rangle$ $\subseteq F$ then by Proposition $5, \phi$ is an automorphism. So let $a_{1} \phi \notin F$ and $a_{2} \phi \notin F$. By Lemma $2 w \phi \sim\langle w\rangle$. We may apply Proposition 10 with $z=w$, $u=a_{1} \phi, v=a_{2} \phi$. Thus there exists an inner automorphism $\psi$ such that $\left\langle a_{1} \phi \psi, a_{2} \phi \psi\right\rangle \subseteq F$. By Proposition 5, $\phi \psi$ is an automorphism and hence $\phi$ is an automorphism. Q.E.D.

Our results enable us to say something about the automorphism group of $G$. Let $S=\left\{\mu \in\right.$ Aut $\left.F: w \mu=w^{e}, \varepsilon= \pm 1\right\} . S$ is the group of all automorphisms $\mu$ of $F$ such that $\langle w \mu\rangle=\langle w\rangle$.

Lemma A. Let $\mu \in S$. Then the mapping $\mu^{*}: G \rightarrow G$ defined by

$$
\begin{aligned}
\mu^{*}: \quad & x \rightarrow x \mu, \quad x \in F, \\
t & \rightarrow t
\end{aligned}
$$

is an automorphism of $G$.

Proof. It is easy to see that $\left(t^{-1} w^{l} t\right) \mu^{*}=\left(w^{m}\right) \mu^{*}$. Since $\mu^{*}$ is clearly surjective it is an automorphism. Q.E.D.

LEMMA B. $S$ is embedded in Aut $G$. 
Proof. The map sending $\mu \rightarrow \mu^{*}$ as above is easily seen to be an embedding. Q.E.D.

Lemma C. Let $\phi \in$ Aut $G$ be such that $F \phi \subseteq F$. Then there exists $x \in F$ such that

(i) $x^{-1}(w \phi) x=w^{\varepsilon}, \quad \varepsilon= \pm 1$,

(ii) $x^{-1}(t \phi) x=t w^{p}$.

PROoF. This is proved by an easy adaptation of the proof of Proposition 5. Q.E.D.

Lemma D. Let $\phi \in$ Aut $G$ be such that $F \phi \subseteq F$ and $w \phi=w^{\varepsilon}, \varepsilon= \pm 1$. Then $F \phi=F$.

Proof. Suppose not; then, say, $a_{1} \notin F \phi$. Since $\phi$ is surjective and $t \phi=$ $w^{p_{0} w^{p_{1}}}$ it is clear that $a_{1} \in\langle F \phi, w, t\rangle$. We claim that $a_{1} \in\langle F \phi, w\rangle$.

Let $a_{1}=y_{0} t^{\eta_{1} y_{1}} \cdots t^{\eta_{y}} y_{s}$ where $y_{i} \in\langle F \phi, w\rangle, i=0,1,2, \ldots, s$. If $s=0$, then there is nothing to prove. If $s>0$, then the right-hand side is not $t$-reduced. So for some $i, t^{\eta_{y_{i}}} t^{\eta_{i+1}} \in\left\langle w^{l\left[-\eta_{i}\right]}\right\rangle$. By induction, $a_{1} \in\langle F \phi, w\rangle$.

However, $w=w^{e} \phi, \varepsilon= \pm 1$, and thus $w \in F \phi$. This is contradictory. Q.E.D.

Write Inn $G$ for the group of inner automorphisms of $G$.

Proposition E. Let $\phi \in$ Aut $G$. Then there exists $\psi \in \operatorname{Inn} G$ such that

(i) $F \phi \psi=F$,

(ii) $w \phi \psi=w^{e}, \varepsilon= \pm 1$,

(iii) $t \phi \psi=-w^{p}$, some $p \in \mathbf{Z}$.

Proof. This is immediate from Proposition 9 and the Lemmas just above. Q.E.D.

We can now describe Aut $G$.

Let $S^{*}$ be the copy of $S$ embedded in Aut $G$. Let $\sigma: G \rightarrow G$ be the automorphism of $G$ defined by

$$
\begin{aligned}
\sigma^{*}: & x \rightarrow x, \quad x \in F, \\
t & \rightarrow t w .
\end{aligned}
$$

Then Aut $G=\left\langle S^{*}, \sigma, \operatorname{Inn} G\right\rangle$. For let $\phi \in$ Aut $G$; let $\psi$ be such that (i), (ii) and (iii) of Proposition E are satisfied. Let $\mu$ be the restriction to $F$ of $\phi \psi$. Then $\phi \psi=\mu^{*} \sigma^{p}$.

We note the following facts:

(a) $\langle\sigma\rangle$ is infinite cyclic.

(b) $\left\langle S^{*}, \sigma\right\rangle=S^{*} \times\langle\sigma\rangle$, the direct product of $S^{*}$ and $\langle\sigma\rangle$.

(c) Inn $G \cong G$ since $G$ has trivial centre. 
(d) $\left\langle S^{*}, \sigma\right\rangle \cap \operatorname{Inn} G=\left\langle\beta^{l}\right\rangle$ where $\beta$ is the inner automorphism of $G$ corresponding to $w$.

To see (d), let $\phi \in\left\langle S^{*}, \sigma\right\rangle \cap \operatorname{Inn} G$. If $\phi=\mu^{*} \sigma^{p}$, then for some $v \in G$ we have $v^{-1} t v=t w^{p}$ and also $v^{-1} w v=w^{\varepsilon}, \varepsilon= \pm 1$. If $|l|,|m| \neq 1$, the latter immediately yields $v \in\langle w\rangle$ and so $\phi \in\langle\beta\rangle$. Then the first of the two equalities yields $v \in\left\langle w^{l}\right\rangle$. Since $\beta^{l}=\left(\gamma^{*}\right)^{l} \sigma^{l-m}$ where $\gamma$ is the inner automorphism of $F$ corresponding to $w$, we have the desired conclusion.

Suppose that $l=1$; then the equation $v^{-1} w v=w^{e}$ yields only the fact $v$ is

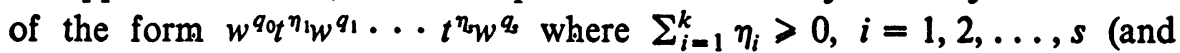
possibly $s=0$ ). However, a simple induction argument shows that this is inconsistent with $v^{-1} t v=t w^{p}$ unless $s=0$. Then we can argue as before.

In conclusion we remark that our description of Aut $G$ is only a relative one since it depends on knowledge of $S$. J. McCool has proved in [6] that $S$ is always finitely presented. Thus we know that Aut $G$ is always finitely generated. It remains to be seen whether or not Aut $G$ is also finitely related.

\section{REFERENCES}

1. G. Baumslag, Residually finite one-relator groups, Bull. Amer. Math. Soc. 73 (1967), 618-620. MR 35 \#2953.

2. G. Baumslag and D. Solitar, Some two-generator, one-relator non-Hopfian groups, Bull. Amer. Math. Soc. 68 (1962), 199-201. MR 26 \#204.

3. G. Baumslag and A. Steinberg, Residual nilpotence and relations in free groups, Bull. Amer. Math. Soc. 70 (1964), 283-284. MR 28 \# 1229.

4. I. M. S. Dey and H. Neumann, The Hopf property of free products, Math. Z. 117 (1970), 325-339. MR 43 \#2099.

5. W. Magnus, A. Karrass and D. Solitar, Combinatorial group theory, Interscience, New York, 1966. MR 34 \#7617.

6. J. McCool, Some finitely presented subgroups of the automorphism group of a free group, J. Algebra 35 (1975), 205-213.

7. S. Meskin, Nonresidually finite one-relator groups, Trans. Amer. Math. Soc. 164 (1972), 105-114. MR 44 \#2807.

8. C. F. Miller III, On group-theoretic decision problems and their classification, Ann. of Math. Studies, no. 68, Princeton Univ. Press, Princeton N. J.; Univ. of Tokyo Press, Tokyo, 1971. MR 46 \#147.

Department of Pure.Mathematics, Queen Mary College, London, England 